\title{
EXTRAGALACTIC CS SURVEY
}

\author{
E. Bayet ${ }^{1}$, R. Aladro ${ }^{2}$, S. Martín ${ }^{3}$, S. Viti ${ }^{1}$, and J. Martín-Pintado ${ }^{4}$ \\ ${ }^{1}$ Department of Physics and Astronomy, University College London, Gower Street, London WC1E 6BT, UK; eb@ star.ucl.ac.uk \\ ${ }^{2}$ IRAM-Instituto de Radioastronomía Milimétrica, Avda. Divina Pastora 7 Local 20, E-18012, Granada, Spain \\ ${ }^{3}$ Harvard Smithsonian Center for Astrophysics, 60 Garden Street, Cambridge, MA 02138, USA \\ ${ }^{4}$ Departamento de Astrofisica Molecular e Infrarroja-Instituto de Estructura de la Materia-CSIC, C Serrano 121, E-28006 Madrid, Spain \\ Received 2009 June 12; accepted 2009 October 20; published 2009 November 18
}

\begin{abstract}
We present a coherent and homogeneous multi-line study of the CS molecule in nearby $(D<10 \mathrm{Mpc})$ galaxies. We include, from the literature, all the available observations from the $J=1-0$ to the $J=7-6$ transitions toward NGC 253, NGC 1068, IC 342, Henize 2-10, M 82, the Antennae Galaxies, and M 83. We have, for the first time, detected the CS(7-6) line in NGC 253, M 82 (both in the northeast and southwest molecular lobes), NGC 4038, M 83 and tentatively in NGC 1068, IC 342, and Henize 2-10. We use the CS molecule as a tracer of the densest gas component of the interstellar medium in extragalactic star-forming regions, following previous theoretical and observational studies by Bayet et al. In this first paper out of a series, we analyze the CS data sample under both local thermodynamical equilibrium (LTE) and non-LTE (large velocity gradient) approximations. We show that except for M 83 and Overlap (a shifted gas-rich position from the nucleus NGC 4039 in the Antennae Galaxies), the observations in NGC 253, IC 342, M 82-NE, M 82-SW, and NGC 4038 are not well reproduced by a single set of gas component properties and that, at least, two gas components are required. For each gas component, we provide estimates of the corresponding kinetic temperature, total CS column density, and gas density.
\end{abstract}

Key words: galaxies: nuclei - ISM: individual (NGC 253, NGC 1068, IC 342, Henize 2-10, M 82, The Antennae, M 83) - ISM: molecules - methods: data analysis - submillimeter

\section{INTRODUCTION}

Detection of star-forming gas is one of the most direct ways to measure the star formation rate and activity in a galaxy, allowing us to significantly improve our understanding of galaxy formation and evolution. Star-forming regions of very dense gas $\left(n\left(\mathrm{H}_{2}\right)>10^{5} \mathrm{~cm}^{-3}\right)$ are needed to maintain star formation activity, even in hostile environments associated with young massive stars. In these environments, star-forming regions are able to resist the disruptive forces (winds or radiation) from nearby newly formed stars longer than the local gas in the local interstellar medium (Klein et al. 1983; Larosa 1983). Determining the physical conditions of the very dense gas in which massive stars form is, thus, essential. In this paper, we study such dense gas and estimate its properties over a large range of nearby galaxy types.

Following theoretical studies by Bayet et al. (2008b) and the first detections of extragalactic very dense gas presented in Mauersberger \& Henkel (1989), Mauersberger et al. (1989), Walker et al. (1990), Martín et al. (2005, 2006a, 2006b), Bayet et al. (2008a), and Greve et al. (2009), we have carried out multiline observations of the CS molecule in nearby $(D<10 \mathrm{Mpc})$ extragalactic environments, enhancing significatively the current data set of extragalactic CS observations. Indeed, CS lines have not been observed so far but in few brightest nearby nuclei such as NGC 253, IC 342, and M 82 and mainly in their lower- $J$ rotational levels (see references above). Hence, the physical properties of such galaxies, namely the kinetic temperature, gas density, etc., were estimated using only a small sample of CS lines. Gas traced by higher- $J$ species, such as CS, has not been characterized so far. In addition, even for the brightest sources (e.g., NGC 253 and M 82), no study of the various velocity components or various positions in the same galaxy has been performed so far in a systematic way. In this paper, first of a series, we thus aim at investigating in much more details the very dense gas properties in many extragalactic sources as traced by the CS molecule.

Sulfur-bearing species are shown to be particularly enhanced during massive star formation, while species such as $\mathrm{HCN}$, although a useful dense gas tracer, may not be tracing the sites where star formation occurs (Lintott \& Viti 2006). Recently, Martín et al. (2005) showed that sulfur emission in the nuclear region of the nearby starburst galaxy NGC 253 is very strong. Among sulfur-bearing species, the CS molecule appears as one of the best tracers of dense gas, especially its high- $J$ rotational transitions such as the CS $J=5-4$ line with an excitation threshold higher than $10^{4}-10^{5} \mathrm{~cm}^{-3}$ (Bronfman et al. 1996) and the CS $J=7-6$ line with a critical density of $n_{\text {crit }} \sim 2 \times 10^{7} \mathrm{~cm}^{-3}$ (Plume et al. 1992).

Selection of nearby sources where we have proposed to observe the CS lines was made through a comparison with past and recent CS observations (see references above). Sources where no CS detections were available from the literature (e.g., Henize 2-10) were chosen on the basis of the detections of the $\mathrm{CO}(6-5)$ or $\mathrm{CO}(7-6)$ lines since these lines are also good tracers of a relatively dense and quite warm gas (Bayet et al. 2004, 2006). The source selection also aimed at presenting various nearby galaxy types where CS line emission can be studied and compared. This sample is far from being unbiased but gathers so far the most complete CS data sample ever obtained in extragalactic sources.

Thus, the centers of NGC 253, IC 342, Henize 2-10, the two molecular lobes of M 82 (northeast and southwest positions), three positions in the Antennae Galaxies (the two nuclei: NGC 4038 and NGC 4039, and a shifted position from NGC 4039 called Overlap), and the center of M 83 were selected, covering a large range of galaxy types and star formation activities (starburst, irregular, merging galaxies, more quiet star-forming galaxies, etc.). We have also included in our source sample the center of NGC 1068 (active galactic nucleus (AGN)-dominated 


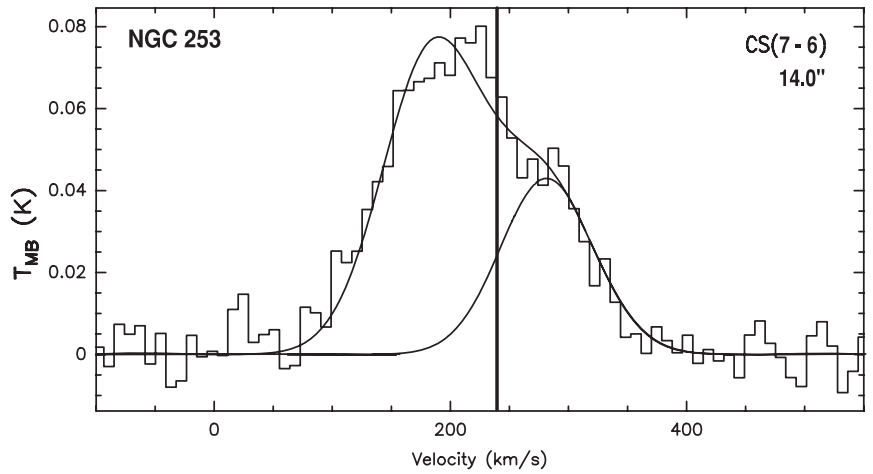

Figure 1. CS(7-6) spectrum in the center of NGC 253. The angular resolution is shown in the upper right corner. The thick vertical black line symbolizes the $V_{\text {LSR }}$ of the source. For NGC 253, we have applied a two-components fit (thin black lines) after having smoothed the entire signal to a velocity resolution of $\approx 10 \mathrm{~km} \mathrm{~s}^{-1}$. The fit on the left is for the emission from NGC 253-1, while on the right it is for the NGC 253-2 emission.

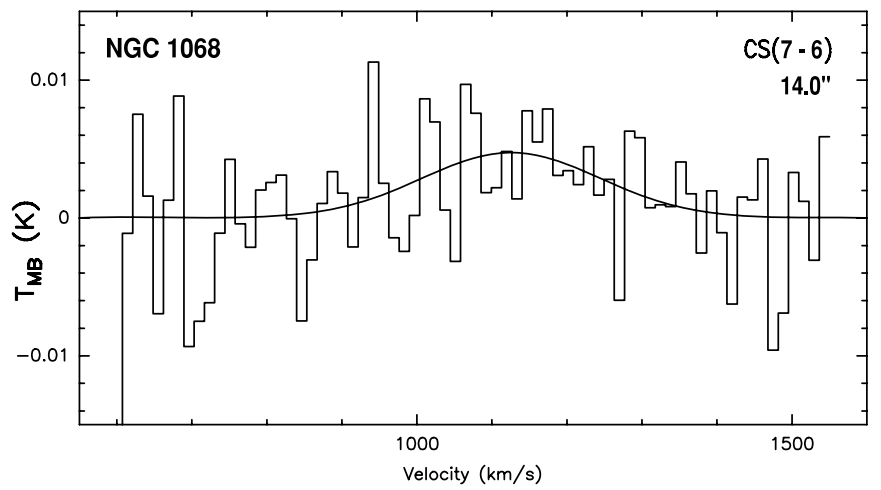

Figure 2. Marginal detection of the CS(7-6) line in the center of NGC 1068 $\left(\Delta v=13.7 \mathrm{~km} \mathrm{~s}^{-1}\right)$.

galaxy), where HCN has been detected (Planesas et al. 1991; Krips et al. 2008).

We present our observations, data reduction, and results in Section 2. In Section 3, we analyze the data set both under local thermodynamical equilibrium (LTE) and non-LTE (large velocity gradient-LVG) approximations (see Sections 3.1 and 3.2). In Sections 4 and 5, we discuss our findings and conclude, respectively.

\section{OBSERVATIONS AND RESULTS}

\subsection{Observational Parameters}

The observations have been performed using the IRAM-30m telescope for the CS(2-1), CS(3-2), and CS(4-3) lines $(v=$ 97.980 GHz, $146.969 \mathrm{GHz}$, and $195.954 \mathrm{GHz}$, respectively) and the James Clerk Maxwell Telescope (JCMT) for the detection of the CS(7-6) transition $(v=342.883 \mathrm{GHz})$. Every $2-3 \mathrm{hr}$, when using both telescopes, the pointing, focus and calibration were performed carefully on planets (Mars and Jupiter) and on evolved stars. The pointing error was estimated to be $\leqslant 3^{\prime \prime}$ in both cases. We have observed the $\operatorname{CS}(5-4)$ line with both telescopes but we have not averaged the signals because the weather conditions were significantly different. Between the two telescope observational sessions, for all the sources but NGC 4038, we have kept the less noisy spectrum (i.e., the spectrum showing the highest signal-to-noise ratio $(\mathrm{S} / \mathrm{N})$ ). These (reduced) spectra are shown in Figures 1-10 and their Gaussian fitting parameters are seen in Table 1. Unfortunately,

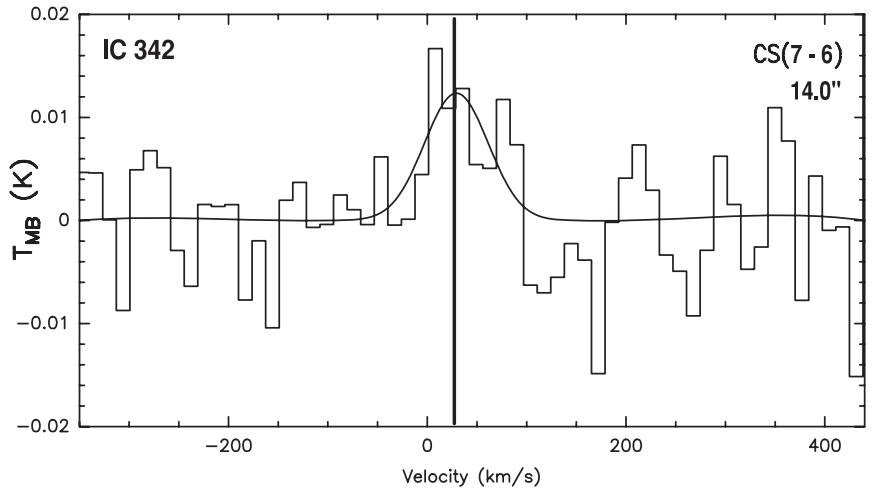

Figure 3. Marginal detection of the CS(7-6) line in the center of IC 342 $\left(\Delta v=13.7 \mathrm{~km} \mathrm{~s}^{-1}\right)$.

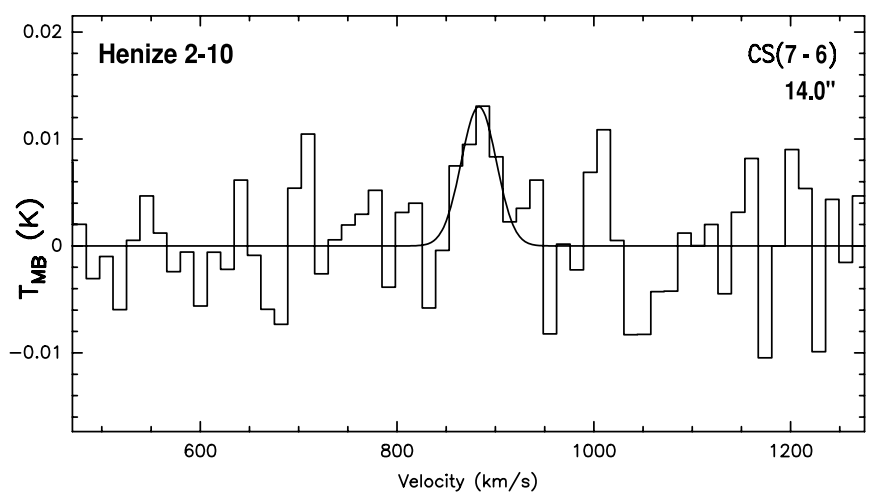

Figure 4. Marginal detection of the CS(7-6) line in the center of Henize 2-10 $\left(\Delta v=13.7 \mathrm{~km} \mathrm{~s}^{-1}\right)$.

for NGC 4038, the reduced spectra obtained with the IRAM$30 \mathrm{~m}$ and with the JCMT are equally noisy (i.e., they have similar $\mathrm{S} / \mathrm{N}$ ) and thus we present both spectra in Figure 7.

The IRAM-30m telescope observations were carried out in 2008 June under various weather conditions $(\approx 1.5-6 \mathrm{~mm}$ of water vapor). Scans were taken using the wobbling secondary with a throw of $120^{\prime \prime}$ for the two positions in M 82 (NE and SW components), while for the Antennae positions (NGC 4038, NGC 4039, and Overlap) we have used the position switching mode with a larger throw of $1^{\prime}$ (reference positions are indicated in Columns 12 and 13 of Table 1). The $100 \mathrm{GHz}$, $150 \mathrm{GHz}, 230 \mathrm{GHz}$, and $270 \mathrm{GHz}$ receivers (A/B/C/D) were used under several configurations, optimizing the allocated time. The $1 \mathrm{MHz}$ and $4 \mathrm{MHz}$ backends were used providing velocity resolution between $1.2 \mathrm{~km} \mathrm{~s}^{-1}$ (at $244 \mathrm{GHz}$ ) and $3.0 \mathrm{~km} \mathrm{~s}^{-1}$ (at $98 \mathrm{GHz}$ ), and $4.9 \mathrm{~km} \mathrm{~s}^{-1}$ (at $244 \mathrm{GHz}$ ) and $12.3 \mathrm{~km} \mathrm{~s}^{-1}$ (at $98 \mathrm{GHz}$ ), respectively. At the observed frequencies of $97.980 \mathrm{GHz}, 146.969 \mathrm{GHz}, 195.954 \mathrm{GHz}$, and $244.936 \mathrm{GHz}$, the IRAM-30m has an HPBW of $\approx 25^{\prime \prime}, 17^{\prime \prime}, 13^{\prime \prime}$, and $10^{\prime \prime}$ with main beam efficiencies of $0.75,0.69,0.65$, and $0.52,{ }^{5}$ respectively. The system temperature ranged from $\approx 200 \mathrm{~K}$ to $\approx 1800 \mathrm{~K}$, depending on the frequency and the weather conditions. The data reduction was performed using the CLASS program of the GILDAS package developed at IRAM.

We have used the JCMT in 2007 March, 2008 February, 2008 July, and 2009 February under medium weather conditions $\left(\tau_{225} \approx 0.15\right)$. We have either used a beam switch mode with a throw of $180^{\prime \prime}$ or a position switch mode with hard offsource reference, depending on the source. We have used the

\footnotetext{
5 See IRAM-30m Web site:

http://www.iram.es/IRAMES/mainWiki/AbcdforAstronomers
} 


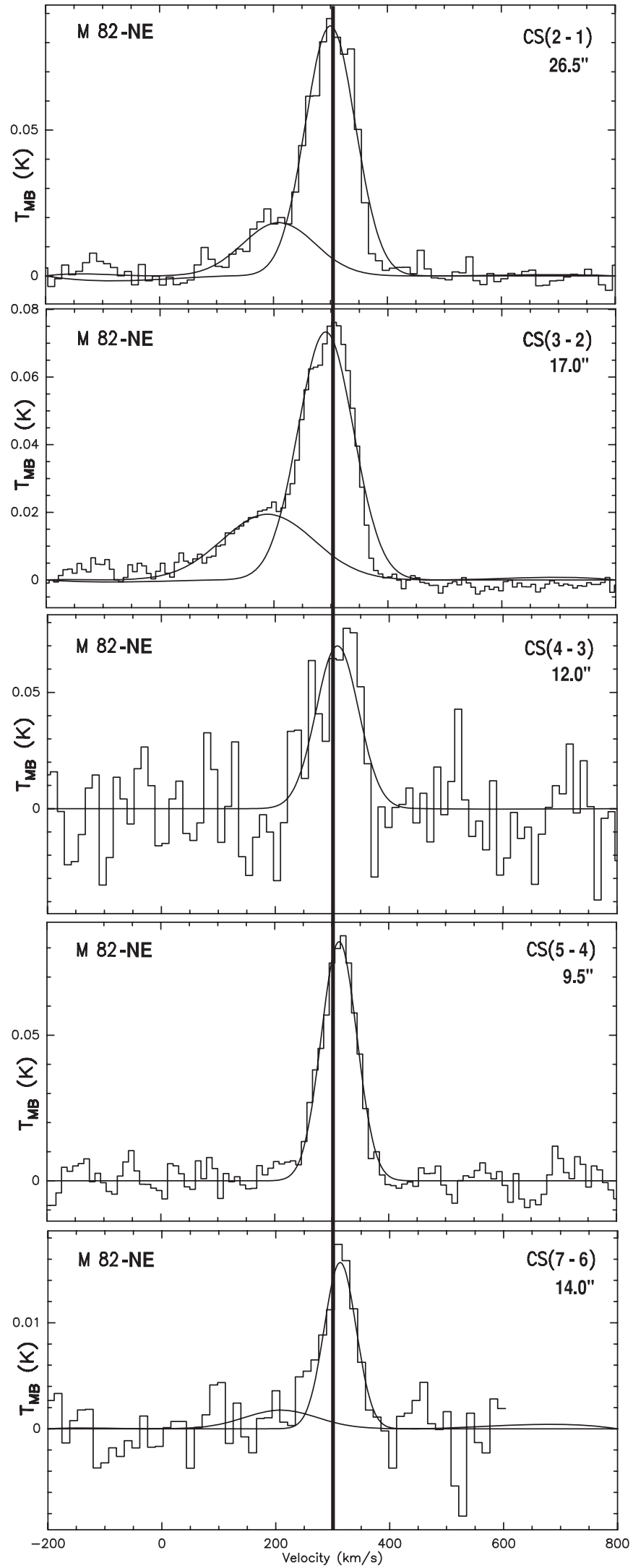

Figure 5. CS spectra in the NE molecular lobe of M 82. The velocity resolution from top to bottom is $\Delta v=12.2 \mathrm{~km} \mathrm{~s}^{-1}, 8.1 \mathrm{~km} \mathrm{~s}^{-1}, 24.5 \mathrm{~km} \mathrm{~s}^{-1}, 9.7 \mathrm{~km} \mathrm{~s}^{-1}$ and $13.7 \mathrm{~km} \mathrm{~s}^{-1}$, respectively.

RxA3 and the 16-pixel camera HARP receivers for the detection of the $\operatorname{CS}(5-4)$ and the $\operatorname{CS}(7-6)$ transitions, respectively. The

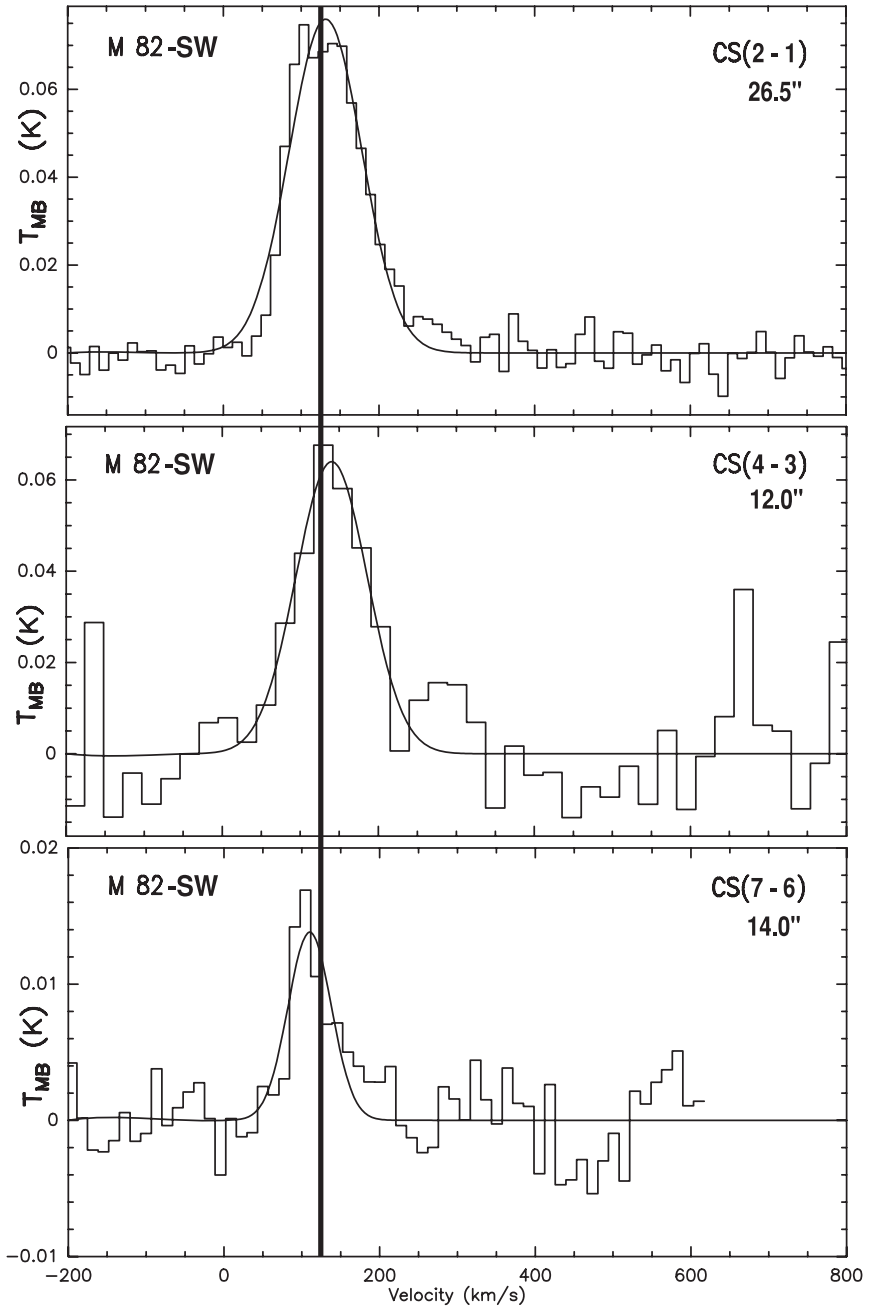

Figure 6. CS spectra in the SW molecular lobe of M 82. The velocity resolution from top to bottom is $\Delta v=12.2 \mathrm{~km} \mathrm{~s}^{-1}, 24.5 \mathrm{~km} \mathrm{~s}^{-1}$, and $13.7 \mathrm{~km} \mathrm{~s}^{-1}$, respectively.

ACSIS digital autocorrelation spectrometer with a bandwidth of $1000 \mathrm{MHz}$ was used at both frequencies and in all the sources because the lines were expected to be broad (see Figures 1-10). The HPBW and the main beam efficiencies of the JCMT at $v=244 \mathrm{GHz}$ and $v=343 \mathrm{GHz}$ are $20^{\prime \prime}$, $14^{\prime \prime}$ and $0.69,0.63,{ }^{6}$ respectively (see Table 1 ). The system temperatures ranged between $200 \mathrm{~K}$ and $400 \mathrm{~K}$. The data prereduction was done using Starlink softwares (KAPPA, SMURF, and STLCONVERT packages) and subsequently translated to CLASS format for final reduction.

All the spectra obtained are shown in Figures 1-10, while the Gaussian fit parameters are listed in Table 1. In this table, we have also included detections of CS lines from the literature (see references mentioned in the last column of the table). Hence this table contains the most complete compilation of CS transitions observed in external galaxies.

\subsection{Results}

For each source, we find a general good agreement between the Gaussian fits and the observations (see Figures 1-10). Table 1 also shows that the velocity positions of the lines and the line widths of the CS transitions are consistent among each other and with previous data from the literature.

\footnotetext{
6 See JCMT Web site: http://www.jach.hawaii.edu/JCMT/instruments/.
} 


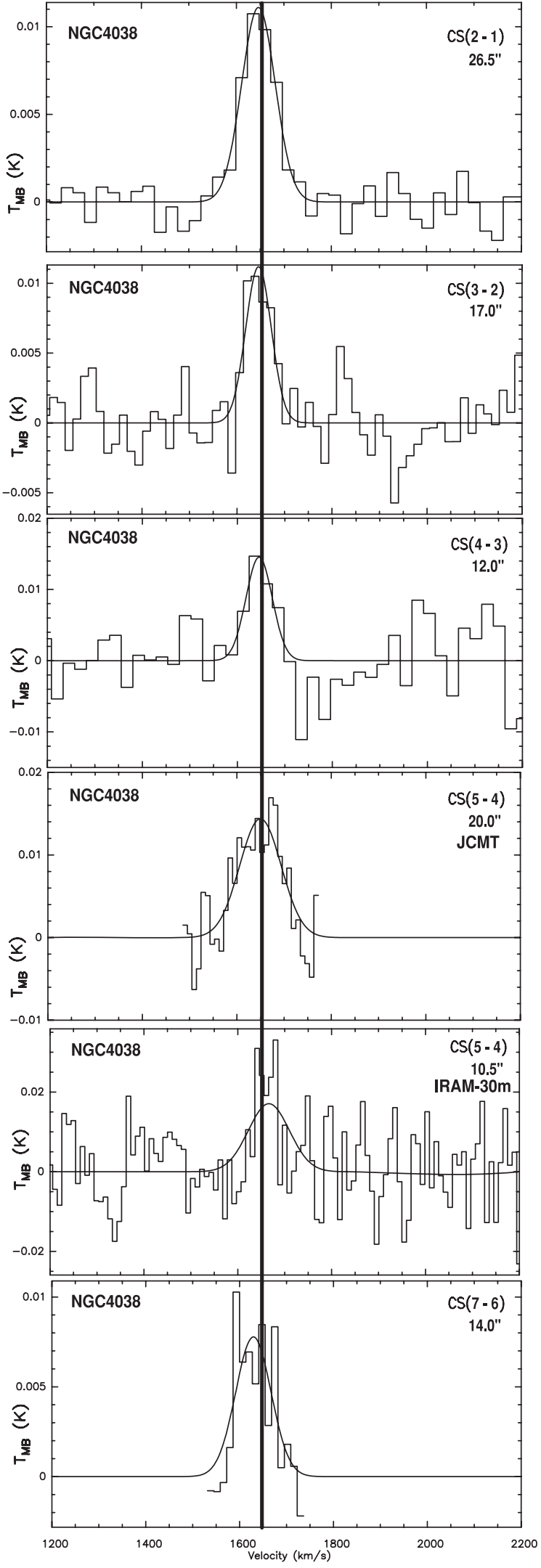

Figure 7. CS spectra in NGC 4038 (one of the nuclei in The Antennae Galaxies). The velocity resolution from top to bottom is $\Delta v=24.5 \mathrm{~km} \mathrm{~s}^{-1}, 16.3 \mathrm{~km} \mathrm{~s}^{-1}$, $24.5 \mathrm{~km} \mathrm{~s}^{-1}, 10.0 \mathrm{~km} \mathrm{~s}^{-1}, 10.0 \mathrm{~km} \mathrm{~s}^{-1}$, and $13.7 \mathrm{~km} \mathrm{~s}^{-1}$, respectively.

For some sources, some lines of CS are only marginally detected. For instance, the CS(7-6) line in NGC 1068 (Figure 2) and in IC 342 (Figure 3) have both an $\mathrm{S} / \mathrm{N}$ lower than 3

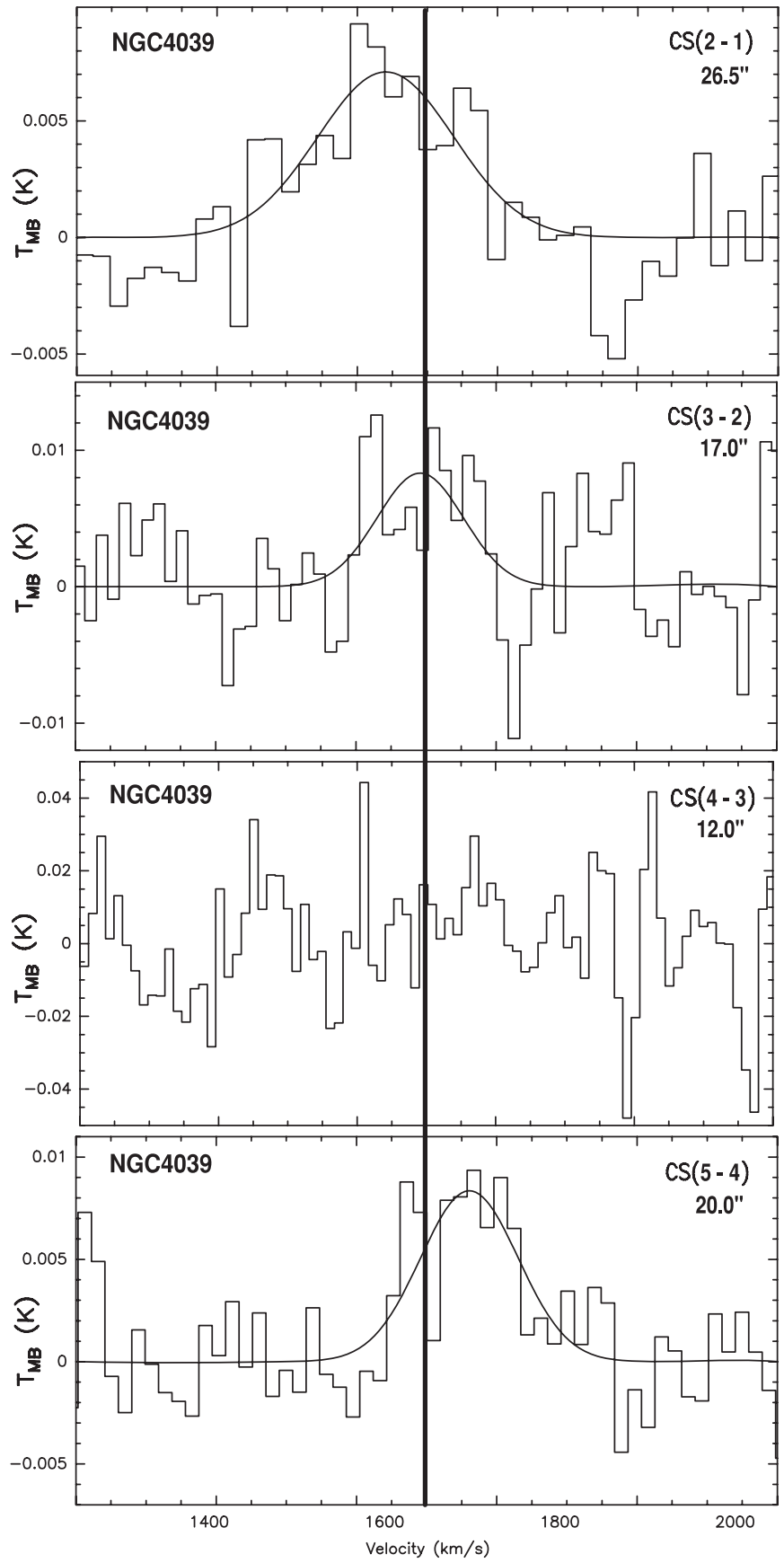

Figure 8. CS spectra in NGC 4039 (one of the nuclei in The Antennae Galaxies) The velocity resolution from top to bottom is $\Delta v=24.5 \mathrm{~km} \mathrm{~s}^{-1}, 16.3 \mathrm{~km} \mathrm{~s}^{-1}$, $12.2 \mathrm{~km} \mathrm{~s}^{-1}$, and $19.1 \mathrm{~km} \mathrm{~s}^{-1}$, respectively.

and thus cannot be considered as detections. We have also obtained for NGC 4039 a hint of a marginal detection, but due to the uncertainty on the velocity position of the line we have not shown the spectrum. Except for NGC 4039, in all the marginal cases presented here, the fitted line width and velocity position match those expected either from lower- $J$ CS lines (when available) or in the case where no other CS lines are available, from other molecular lines such as $\mathrm{CO}$ as in the case of the CS(7-6) line in Henize 2-10. For this source, we have derived an upper limit of the source-averaged CS column density of $N(\mathrm{CS})=7.3 \times 10^{12} \mathrm{~cm}^{-2}$ from the CS(7-6) marginal detection, using a source size of 13" (see Meier et al. 2001; Bayet et al. 2004). Due to the fact that there is only one CS observation 


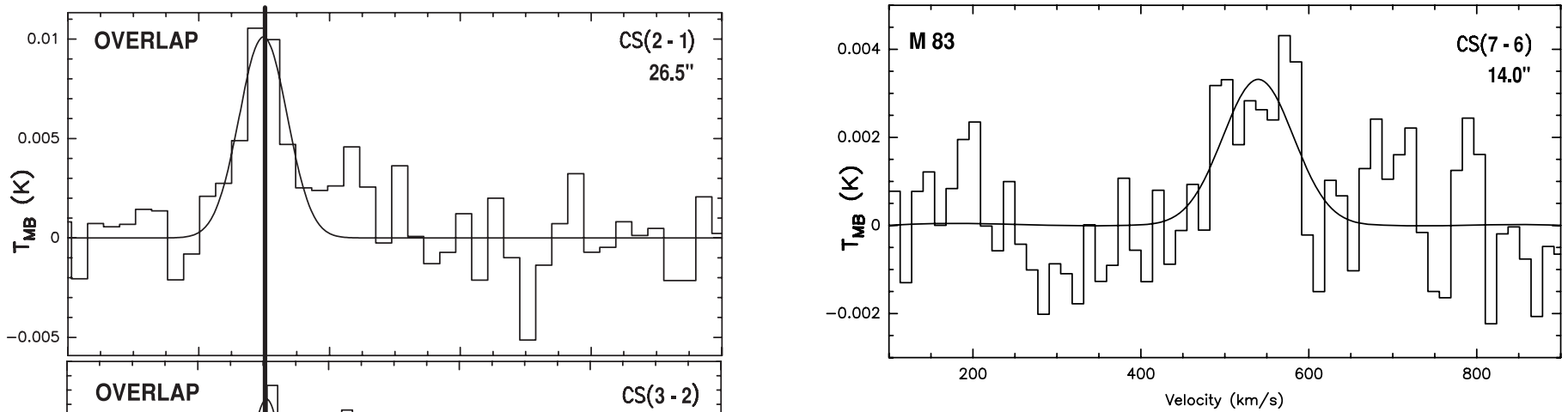

Figure 10. CS(7-6) spectrum in the center of M $83\left(\Delta v=13.7 \mathrm{~km} \mathrm{~s}^{-1}\right)$.

For NGC 253, based on the literature, it is expected that some CS detections show a double peak emissions corresponding to two gas components (see Martín et al. 2005; Figure 1). Thus, in NGC 253, we have called NGC 253-1 the gas component whose line emission is located at a velocity position of about 180-200 $\mathrm{km} \mathrm{s}^{-1}$, while NGC 253-2 corresponds to the higher velocity gas component located at about $280-300 \mathrm{~km} \mathrm{~s}^{-1}$. A two-components Gaussian fit has thus been applied. We have listed the fit parameters obtained in Table 1.

In some of the M 82-NE CS spectra (see Figure 5), one also notice a double peak emission. However, the nature of this emission differs significantly from the one observed in NGC 253. Indeed, after having compared Figure 5 with the data from Bayet et al. (2008a), we have concluded that the weaker peak (seen on the left side of the spectra) corresponds to a contamination from the nucleus (partially included into the telescope beam). We have thus excluded such emission from the analysis performed in Sections 3.1 and 3.2.

\section{MOLECULAR EXCITATION}

\subsection{LTE Analysis}

To obtain an estimation of the kinetic temperature and the total CS column density in the various extragalactic star-forming regions we survey here, we have used first the rotational diagram method, corrected for beam dilution effects and assuming optically thin emission (antennae temperature proportional to the column density in the upper level of the observed transition). The beam dilution effect has been removed assuming a source size of $20^{\prime \prime}, 20^{\prime \prime}, 4^{\prime \prime}, 6^{\prime \prime}, 6^{\prime \prime}, 8^{\prime \prime} .5,7^{\prime \prime}, 5^{\prime \prime} .5,20^{\prime \prime}$, and $5^{\prime \prime}$ for NGC 253-1, NGC 253-2, NGC 1068, IC 342, M82-NE, M 82SW, NGC 4038, NGC 4039, Overlap, and M 83, respectively. These values have been selected from the available maps of CS published in the literature or from maps of other tracers of dense gas. When neither was available, we have used, by default, interferometric CO maps. Namely, for the NGC 253 source size, we refer to the CS(2-1) map of Peng et al. (1996), in agreement with the values used for $\mathrm{SO}_{2}, \mathrm{NS}$, and $\mathrm{NO}$ emission (tracers of relatively dense gas) presented in Martín et al. (2003). For NGC 1068, we have used the HCN maps from Helfer \& Blitz (1995) and Krips et al. (2008) for deriving the CS emitting source size. For IC 342, we base our estimation on the HCN interferometric map of Schinnerer et al. (2008), whereas for M 82, we used $\mathrm{SiO}$ and $\mathrm{HCO}$ interferometric maps from García-Burillo et al. (2001, 2002), respectively. For the three positions in the Antennae Galaxies, we have used the interferometric CO map from Wilson et al. (2000) as an upper limit of the CS emitting source size, while for M 83 we have used the recent $\mathrm{HCN}$ so far (see Figure 4), we have excluded Henize 2-10 from the data analysis presented in Section 3.

Figure 9. CS spectra in the Overlap position (The Antennae Galaxies). Due to poor weather conditions, no detection is seen either in the CS(4-3), or the $\mathrm{CS}(5-4)$ or the $\mathrm{CS}(7-6)$ lines. The velocity resolution from top to bottom is $\Delta v=24.5 \mathrm{~km} \mathrm{~s}^{-1}, 16.3 \mathrm{~km} \mathrm{~s}^{-1}, 24.5 \mathrm{~km} \mathrm{~s}^{-1}, 19.6 \mathrm{~km} \mathrm{~s}^{-1}$, and $13.7 \mathrm{~km} \mathrm{~s}^{-1}$ respectively. 
Table 1

Observational Parameters and Gaussian Fits for the Data Set of CS Observations

\begin{tabular}{|c|c|c|c|c|c|c|c|c|c|c|c|c|c|}
\hline Source & Line & $(\mathrm{GHz})$ & $\begin{array}{l}T_{\text {sys }} \\
(\mathrm{K})\end{array}$ & $\begin{array}{l}\text { Beam } \\
\text { Size } \\
\left({ }^{\prime \prime}\right)\end{array}$ & $\begin{array}{l}\text { Telesc. } \\
\text { Name }\end{array}$ & $\begin{array}{c}\int\left(T_{\mathrm{mb}} d v\right) \\
\left(\mathrm{K} \mathrm{km} \mathrm{s}^{-1}\right)\end{array}$ & $\begin{array}{c}V_{\text {peak }} \\
\left(\mathrm{km} \mathrm{s}^{-1}\right)\end{array}$ & $\begin{array}{l}\text { FWHM } \\
\left(\mathrm{km} \mathrm{s}^{-1}\right)\end{array}$ & $T_{\text {peak }}$ & $(\mathrm{mK})$ & R.A. (J2000) & Decl. (J2000) & References \\
\hline \multirow[t]{5}{*}{ NGC 253-1 } & $\mathrm{CS}(2-1)$ & 97.980 & $\ldots$ & 51.0 & SEST & $4.4 \pm 0.5$ & 172 & 96 & 43.4 & 5.2 & $00: 47: 33.4$ & $-25: 17: 23.0$ & 4 \\
\hline & $\mathrm{CS}(3-2)$ & 146.969 & $\ldots$ & 16.7 & IRAM-30m & $11.9 \pm 0.2$ & 185 & 100 & 111.2 & 4.1 & $"$ & $"$ & 4 \\
\hline & $\mathrm{CS}(4-3)$ & 195.954 & $\ldots$ & 12.6 & IRAM-30m & $11.5 \pm 0.4$ & 185 & 100 & 108.2 & 7.0 & $"$ & " & 4 \\
\hline & $\mathrm{CS}(5-4)$ & 244.936 & $\ldots$ & 21.0 & SEST & $4.4 \pm 0.4$ & 158 & 107 & 38.2 & 10.8 & $"$ & $"$ & 4 \\
\hline & $\operatorname{CS}(7-6)$ & 342.883 & 679 & 14.0 & JCMT & $7.9 \pm 0.3$ & $185^{\mathrm{a}}$ & $110.1 \pm 4.4$ & 67.1 & 5.1 & $"$ & $"$ & 1 \\
\hline \multirow[t]{5}{*}{ NGC 253-2 } & $\mathrm{CS}(2-1)$ & 97.980 & $\ldots$ & 51.0 & SEST & $8.2 \pm 0.5$ & 290 & 116 & 66.5 & 5.2 & $00: 47: 33.4$ & $-25: 17: 23.0$ & 4 \\
\hline & $\mathrm{CS}(3-2)$ & 146.969 & $\ldots$ & 16.7 & IRAM-30m & $13.7 \pm 0.2$ & 288 & 117 & 110.3 & 4.1 & $"$ & $"$ & 4 \\
\hline & $\mathrm{CS}(4-3)$ & 195.954 & $\ldots$ & 12.6 & IRAM-30m & $13.4 \pm 0.5$ & 288 & 121 & 104.5 & 7.0 & $"$ & $"$ & 4 \\
\hline & $\mathrm{CS}(5-4)$ & 244.936 & $\ldots$ & 21.0 & SEST & $5.4 \pm 1.3$ & 262 & 108 & 46.7 & 10.8 & $"$ & $"$ & 4 \\
\hline & $\operatorname{CS}(7-6)$ & 342.883 & 679 & 14.0 & JCMT & $4.4 \pm 0.3$ & $288^{a}$ & $107.1 \pm 7.1$ & 38.7 & 5.1 & $"$ & $"$ & 1 \\
\hline \multirow[t]{3}{*}{ NGC 1068} & $\mathrm{CS}(3-2)$ & 146.969 & 600 & 16.7 & IRAM-30m & $9.1 \pm 1.5$ & 1100 & 245.0 & 30.0 & 10.0 & $02: 42: 40.7$ & $-00: 00: 47.6$ & 5 \\
\hline & $\mathrm{CS}(5-4)$ & 244.936 & $\ldots$ & 10.0 & IRAM-30m & $3.3 \pm 0.3$ & $1125 \pm 9.0$ & $180.0 \pm 20.0$ & 17.6 & 3.0 & $"$ & $"$ & 6 \\
\hline & $\mathrm{CS}(7-6)$ & 342.883 & 307 & 14.0 & JCMT & $1.4 \pm 0.5$ & $1125.4 \pm 49.0$ & $279.0 \pm 99.3$ & 4.8 & 8.1 & $"$ & $"$ & 1 \\
\hline \multirow[t]{5}{*}{ IC 342} & $\mathrm{CS}(1-0)$ & 48.991 & $350-550$ & 36.0 & NRO & $2.5 \pm 0.4$ & 30 & $50-60$ & 50.0 & 12.0 & $03: 46: 48.3$ & $68: 05: 46.0$ & 7 \\
\hline & $\mathrm{CS}(2-1)$ & 97.980 & 188 & 25.1 & IRAM-30m & $5.0 \pm 0.1$ & $31.7 \pm 0.6$ & $53.9 \pm 1.5$ & 87.4 & 2.5 & $"$ & " & 3 \\
\hline & $\mathrm{CS}(3-2)$ & 146.969 & 225 & 16.7 & IRAM-30m & $4.9 \pm 0.1$ & $31.2 \pm 0.6$ & $51.7 \pm 1.6$ & 88.6 & 3.0 & $"$ & $"$ & 3 \\
\hline & $\mathrm{CS}(5-4)$ & 244.936 & 378 & 10.0 & IRAM-30m & $2.5 \pm 0.2$ & $38.2 \pm 2.0$ & $63.4 \pm 5.1$ & 37.6 & 2.9 & $"$ & $"$ & 3 \\
\hline & $\mathrm{CS}(7-6)$ & 342.883 & 525 & 14.0 & JCMT & $1.0 \pm 0.4$ & $29.3 \pm 15.3$ & $75.6 \pm 30.6$ & 12.3 & 8.5 & ” & $"$ & 1 \\
\hline Henize 2-10 & $\mathrm{CS}(7-6)$ & 342.883 & 252 & 14.0 & $\mathrm{JCMT}$ & $0.6 \pm 0.2$ & $883.1 \pm 7.4$ & $42.0 \pm 16.1$ & 13.0 & 7.4 & $08: 36: 15.2$ & $-26: 24: 34.0$ & 1 \\
\hline \multirow[t]{5}{*}{ M 82_NE } & $\mathrm{CS}(2-1)$ & 97.980 & 236 & 25.1 & IRAM-30m & $9.4 \pm 0.2$ & $299.9 \pm 1.3$ & $103.4 \pm 3.3$ & 85.7 & 8.5 & $09: 55: 54.4$ & $69: 40: 54.6$ & 1 \\
\hline & $\mathrm{CS}(3-2)$ & 146.969 & 278 & 16.7 & IRAM-30m & $8.9 \pm 0.1$ & $290.9 \pm 0.7$ & $114.6 \pm 2.0$ & 73.3 & 1.7 & $"$ & $"$ & 1 \\
\hline & $\mathrm{CS}(4-3)$ & 195.954 & 1425 & 12.6 & IRAM-30m & $6.5 \pm 0.8$ & $309.2 \pm 6.1$ & $87.7 \pm 12.1$ & 70.1 & 17.2 & $"$ & $"$ & 1 \\
\hline & $\mathrm{CS}(5-4)$ & 244.936 & 772 & 10.0 & IRAM-30m & $6.4 \pm 0.2$ & $313.0 \pm 1.1$ & $73.5 \pm 2.6$ & 82.1 & 4.8 & $"$ & $"$ & 1 \\
\hline & $\operatorname{CS}(7-6)$ & 342.883 & 309 & 14.0 & JCMT & $1.1 \pm 0.2$ & $313.2 \pm 4.4$ & $58.1 \pm 6.8$ & 15.7 & 2.9 & $"$ & " & 1 \\
\hline \multirow[t]{3}{*}{ M 82_SW } & $\mathrm{CS}(2-1)$ & 97.980 & 237 & 25.1 & IRAM-30m & $8.9 \pm 0.2$ & $131.4 \pm 1.2$ & $109.8 \pm 2.7$ & 75.9 & 3.8 & $09: 55: 49.4$ & $69: 40: 39.6$ & 1 \\
\hline & $\mathrm{CS}(4-3)$ & 195.954 & 1628 & 12.6 & IRAM-30m & $7.3 \pm 1.0$ & $140.0 \pm 6.6$ & $104.7 \pm 17.2$ & 65.5 & 17.9 & $"$ & $"$ & 1 \\
\hline & $\mathrm{CS}(7-6)$ & 342.883 & 340 & 14.0 & JCMT & $1.0 \pm 0.2$ & $110.2 \pm 8.8$ & $60.3 \pm 14.8$ & 13.8 & 5.1 & ” & $"$ & 1 \\
\hline \multirow[t]{6}{*}{ NGC 4038} & $\mathrm{CS}(2-1)$ & 97.980 & 201 & 25.1 & IRAM-30m & $1.0 \pm 0.1$ & $1646.8 \pm 3.0$ & $79.6 \pm 6.7$ & 11.6 & 2.2 & $12: 01: 52.8$ & $-18: 52: 05.3$ & 1 \\
\hline & $\mathrm{CS}(3-2)$ & 146.969 & 471 & 16.7 & IRAM-30m & $0.8 \pm 0.1$ & $1645.2 \pm 3.8$ & $62.2 \pm 7.6$ & 11.4 & 2.6 & " & $"$ & 1 \\
\hline & $\mathrm{CS}(4-3)$ & 195.954 & 1396 & 12.6 & IRAM-30m & $1.1 \pm 0.3$ & $1646.0 \pm 8.6$ & $65.4 \pm 15.8$ & 15.3 & 6.5 & $"$ & $"$ & 1 \\
\hline & $\mathrm{CS}(5-4)$ & 244.936 & 326 & 20.0 & JCMT & $1.6 \pm 0.2$ & $1649.9 \pm 6.5$ & $103.2 \pm 12.7$ & 14.4 & 6.4 & $"$ & $"$ & 2 \\
\hline & $\mathrm{CS}(5-4)$ & 244.936 & 1924 & 10.5 & IRAM-30m & $1.9 \pm 0.4$ & $1666.3 \pm 9.7$ & 103.0 & 17.1 & 9.5 & $"$ & $"$ & 1 \\
\hline & $\operatorname{CS}(7-6)$ & 342.883 & 250 & 14.0 & JCMT & $0.7 \pm 0.1$ & $1629.4 \pm 7.6$ & $88.6 \pm 15.0$ & 7.8 & 2.7 & ” & $"$ & 1 \\
\hline \multirow[t]{4}{*}{ NGC 4039} & $\mathrm{CS}(2-1)$ & 97.980 & 226 & 25.1 & IRAM-30m & $1.7 \pm 0.2$ & $1640.8 \pm 16.3$ & $228.2 \pm 33.6$ & 7.1 & 3.2 & $12: 01: 53.5$ & $-18: 53: 11.3$ & 1 \\
\hline & $\mathrm{CS}(3-2)$ & 146.969 & 547 & 16.7 & IRAM-30m & $1.3 \pm 0.3$ & $1691.6 \pm 19.1$ & $145.0 \pm 30.2$ & 8.3 & 5.8 & $"$ & $"$ & 1 \\
\hline & $\mathrm{CS}(4-3)$ & 195.954 & 1275 & 12.6 & IRAM-30m & & $\ldots$ & $\ldots$ & $\ldots$ & 17.9 & $"$ & $"$ & 1 \\
\hline & $\mathrm{CS}(5-4)$ & 244.936 & 277 & 20.0 & JCMT & $1.4 \pm 0.2$ & $1757.6 \pm 12.7$ & $157.1 \pm 26.5$ & 8.2 & 4.0 & " & , & 1 \\
\hline \multirow[t]{5}{*}{ OVERLAP } & $\mathrm{CS}(2-1)$ & 97.980 & 203 & 25.1 & IRAM-30m & $0.9 \pm 0.2$ & $1498.8 \pm 5.6$ & $81.2 \pm 18.9$ & 10.6 & 2.7 & $12: 01: 54.9$ & $-18: 52: 59.0$ & 1 \\
\hline & $\mathrm{CS}(3-2)$ & 146.969 & 467 & 16.7 & IRAM-30m & $0.8 \pm 0.2$ & $1504.8 \pm 6.4$ & $61.8 \pm 18.7$ & 11.7 & 4.8 & $"$ & $"$ & 1 \\
\hline & $\operatorname{CS}(4-3)$ & 195.954 & 1290 & 12.6 & IRAM-30m & $\ldots$ & $\ldots$ & $\ldots$ & $\ldots$ & 14.0 & $"$ & $"$ & 1 \\
\hline & $\mathrm{CS}(5-4)$ & 244.936 & 1763 & 10.0 & IRAM-30m & $\ldots$ & $\ldots$ & $\ldots$ & $\ldots$ & 14.4 & $"$ & $"$ & 1 \\
\hline & $\operatorname{CS}(7-6)$ & 342.883 & 230 & 14.0 & JCMT & $\ldots$ & $\ldots$ & $\ldots$ & $\ldots$ & 4.3 & $"$ & $"$ & 1 \\
\hline \multirow[t]{3}{*}{ M 83} & $\mathrm{CS}(3-2)$ & 146.969 & 600 & 16.7 & IRAM-30m & $1.2 \pm 0.3$ & $553.0 \pm 11.0$ & $78.0 \pm 24.0$ & 15.0 & $\ldots$ & $13: 36: 59.2$ & $-29: 52: 04.5$ & 5 \\
\hline & $\mathrm{CS}(5-4)$ & 244.936 & $\ldots$ & 10.0 & IRAM-30m & $2.3 \pm 0.3$ & $504.0 \pm 7.0$ & $82.4 \pm 1.1$ & 26.5 & 0.5 & $"$ & $"$ & 4 \\
\hline & $\mathrm{CS}(7-6)$ & 342.883 & 121 & 14.0 & JCMT & $0.4 \pm 0.1$ & $540.0 \pm 9.5$ & $99.1 \pm 18.8$ & 3.3 & 1.1 & $"$ & $"$ & 1 \\
\hline
\end{tabular}

Notes. Where data were not available, we have put a black dash in the corresponding place in the table.

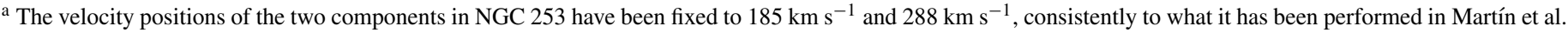
(2005).

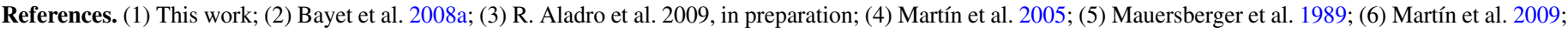
(7) Paglione et al. 1995.

interferometric map of Muraoka et al. (2009). In Figures 11-16, we show the resulting rotational diagrams for all the sources.

The rotational diagram approach (see Goldsmith \& Langer 1999 and appendix in Turner 1991; Girart et al. 2002; Martín et al. 2005) assumes the gas in LTE. If correct, then the data are well fitted by a linear regression using a single component. In fact, assuming LTE in rotational diagrams implies that the rotational temperature is equal to the excitation temperatures of all the observed transitions. As the gas may not be thermalized, the derived rotational temperature is thus a lower limit to the kinetic temperature.

The LTE solutions are shown in Figures $11-16$ by dashed lines (single-component fit). ${ }^{7}$ As clearly seen in the figures, most of the data are not well fitted by a single-component fit.

\footnotetext{
7 The fits have been performed by the xmgrace software.
} 


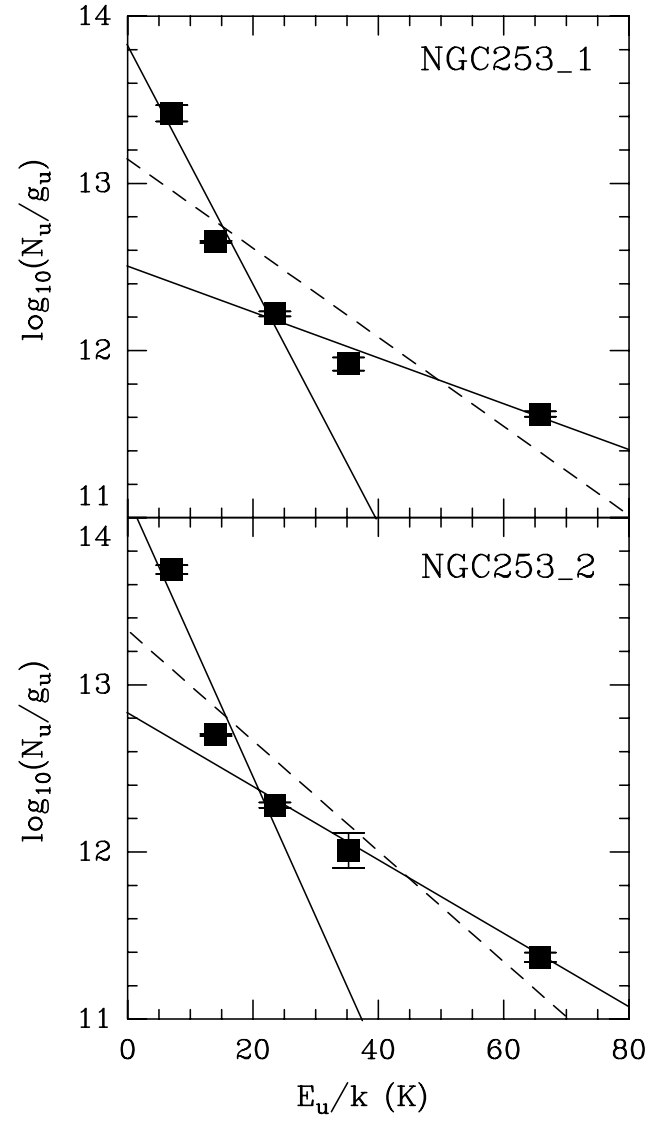

Figure 11. CS rotational diagrams of NGC 253-1 (top plot) and NGC 253-2 (bottom plot). The linear regression using a single component is represented by dashed black lines, while the two-components regression is represented by solid black lines. Detections are represented by black square symbols with error bars. These error bars actually correspond to those of the integrated intensities (order of $10 \%-20 \%$ ). When a CS line is marginally detected (upper limit), we represent the corresponding datum with an open white square (see also Figures 12, 13, and 15).

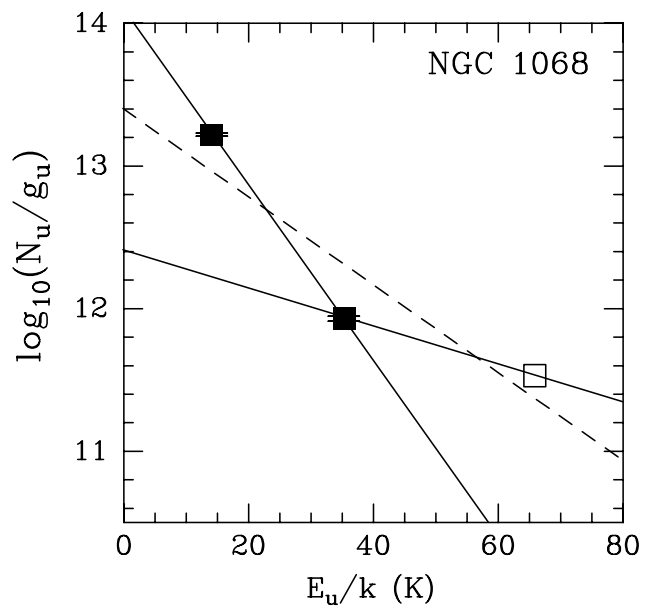

Figure 12. CS rotational diagram of the center of NGC 1068.

This is not surprising since it is expected that the gas contained in the nucleus of galaxies, such as NGC 253, IC 342, etc., is not in thermal equilibrium. Thus, to better reproduce the data, we have adopted a very simple approach assuming that, when the single-component fit is not applicable, at least two gas components exist in the observed regions: one responsible for the low-J CS emission and one for the high-J CS line emissions. The fits performed with two components are shown

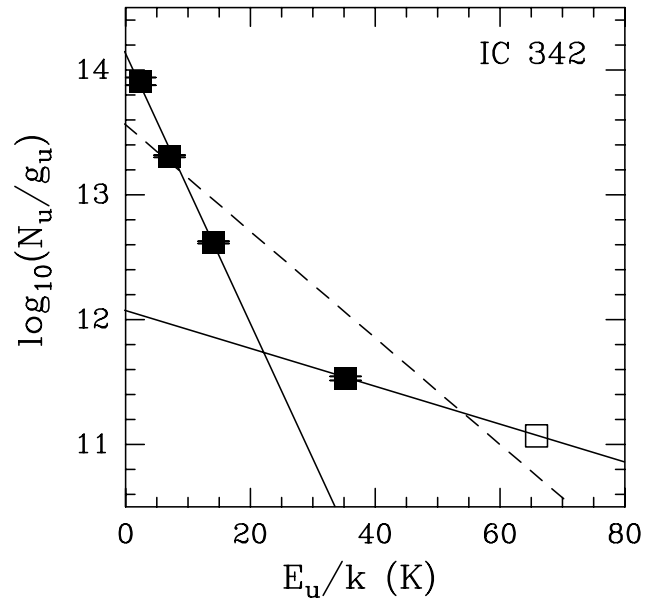

Figure 13. CS rotational diagram of the center of IC 342 .

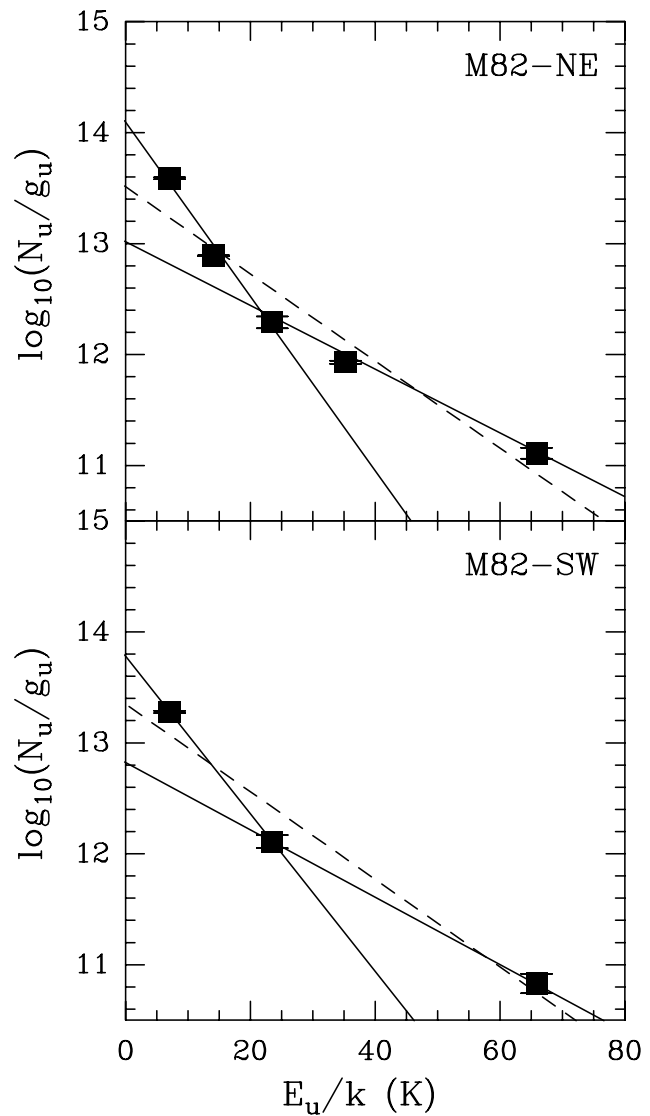

Figure 14. CS rotational diagrams for the M 82-NE (top plot) and the M 82-SW (bottom plot) molecular lobes.

by solid lines in Figures 11-15. Note however that when a source shows two clear detections and an upper limit, our approach is crude. Whereas we simply fitted the rotation diagrams with two temperature components, it is clear that there is a continuum change of the excitation temperature.

The exceptions to this approach are M 83 and the Overlap position. Indeed, for these sources, the CS data appear to be well fitted by a single-component fit. This means that, in these sources, the gas might be more homogeneously distributed than in other sources. This feature has already been observed in $\mathrm{M}$ 83 with the CO data (Bayet et al. 2006). Bayet et al. (2006) indeed showed that the same gas component well reproduce the 


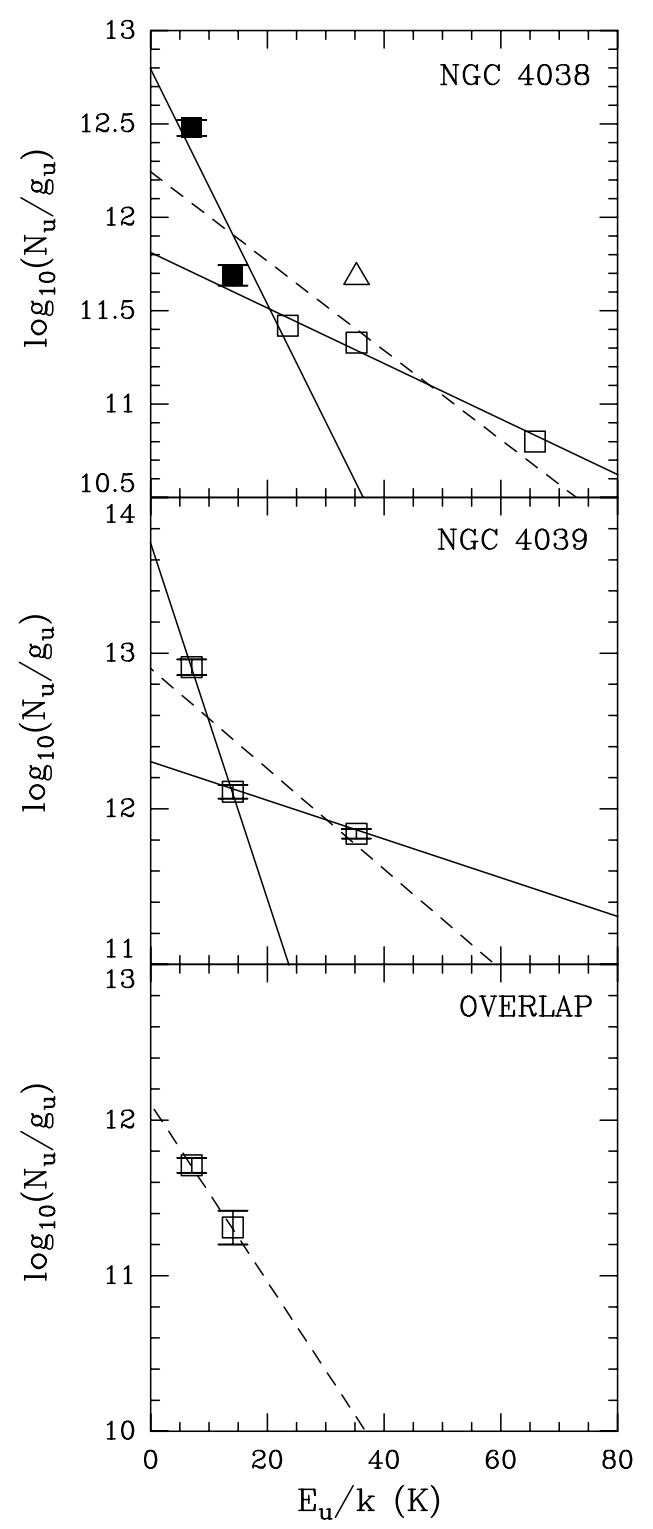

Figure 15. CS rotational diagrams of the three positions observed in the Antennae: NGC 4038 (top plot), NGC 4039 (middle plot), and Overlap (bottom plot). For the NGC 4038 CS(5-4) line, we have used a white open triangle and a white open square for representing the IRAM-30m and the JCMT observations, respectively.

$\mathrm{CO}(3-2), \mathrm{CO}(4-3)$, and $\mathrm{CO}(6-5)$ lines in the center of $\mathrm{M} 83$, the requirement for another (colder) gas component appearing only when considering lower- $J$ CO lines. The current rotational diagram for M 83 and Overlap contains only three and two CS detections, respectively. It may be that the two-components fit may be necessary once the $\mathrm{CS}(1-0), \operatorname{CS}(2-1)$, and $\mathrm{CS}(4-3)$ lines will be observed in M 83 and when the CS(1-0), CS(4-3), CS(5-4), and CS(7-6) transitions will be added to the rotational diagram of Overlap.

In Figures 11-16, we have used different symbols to represent marginal detections (open white squares) and detections (black filled squares). For NGC 4038, two CS(5-4) (marginal) detections exist. We have shown the IRAM-30m one with an open white triangle and the JCMT with an open white square since it appears in better agreement with the rest of the data and shows a higher $\mathrm{S} / \mathrm{N}$ (see Figure 7 and Table 1).

The rotational temperatures we have obtained for each source are listed in Table 2. The typical uncertainties on the rotational

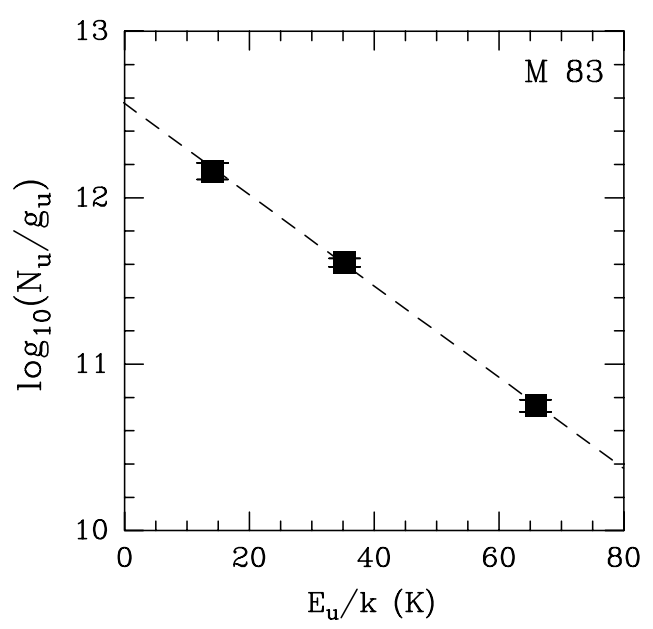

Figure 16. CS rotational diagram of the center of M 83 .

temperatures are of 5\%-6\%. We have also derived the sourceaveraged total column densities $N(\mathrm{CS})$ for each component in each source (see Table 2). For determining such values, we have used the partition function from the CDMS Web site, ${ }^{8}$ extrapolating their values to the range of rotational temperatures listed in Table 2 (linear interpolation applied).

Below, we compare our results with those available in the literature.

1. NGC 253. As expected, the CS column densities for both NGC 253-1 and NGC 253-2 sources (low- and highvelocity components of NGC 253) are in agreement with the values derived by Martín et al. (2005). Indeed, for the NGC 253-1 source, the CS column density we have computed for the single-component fit is in agreement within a factor of 0.9. For the NGC 253-2 source, the CS column density for the single-component fit is in agreement within a factor of 1.8. The differences between these two studies originate clearly from the presence of the new CS(7-6) observations. This transition reveals or confirms in most of the cases the need of at least one more component to better reproduce the data.

2. IC 342. Mauersberger et al. (1989) have also observed in various CS transitions the center of the galaxy IC 342 . They estimated the column density to be $N(\mathrm{CS})=1.5 \times$ $10^{14} \mathrm{~cm}^{-2}$, in agreement (with a factor of 0.5 ) with our estimate (single-component fit).

3. Molecular lobes in M 82. M 82-NE and M 82-SW lobes have never been observed previously in any CS lines. However, Bayet et al. (2008a) showed that in the center of M 82 the CS column density is estimated to be $N(\mathrm{CS})=$ $(0.13-6.7) \times 10^{14} \mathrm{~cm}^{-2}$. This is of the same order of magnitude of the values presented in Table 2 (singlecomponent fit) for the lobe positions.

4. The Antennae Galaxy. Similarly to the lobes in M 82, no CS detection has been published so far neither in NGC 4039 nor in the Overlap position in the Antennae Galaxies. Only for NGC 4038, Bayet et al. (2008a) have obtained $N(\mathrm{CS})=$ $1.8 \times 10^{13} \mathrm{~cm}^{-2}$ (from the single CS(5-4) observation). This estimate is in agreement with the values listed in Table 2 (single-component fit).

5. M 83. The CS column density computed when adding the $\operatorname{CS}(7-6)$ line to the data available from the literature is in

8 See http://www.astro.uni-koeln.de/site/vorhersagen/catalog/partition_function.html. 
Table 2

Results of the Linear Regressions (Slope, Correlation Coefficient, Total Source-averaged CS Column Density, and Rotational Temperature) for a Single- and Two-components Fit

\begin{tabular}{|c|c|c|c|c|}
\hline $\begin{array}{l}\text { Source (No. of } \\
\text { Fit Components) }\end{array}$ & $\begin{array}{c}\text { Slope } \\
\left(\times 10^{-2}\right)\end{array}$ & $\begin{array}{l}\text { Correl. } \\
\text { Coeff. }\end{array}$ & $\begin{array}{l}N(\mathrm{CS})^{\mathrm{a}} \\
\left(\times 10^{14}\right)\end{array}$ & $\begin{array}{l}T_{\text {rot }}{ }^{a} \\
\text { (in K) }\end{array}$ \\
\hline NGC 253-1 (one) & -2.67 & 0.878 & 1.79 & 16.3 \\
\hline \multirow[t]{2}{*}{ NGC 253-1 (two) } & -7.14 & 0.970 & 2.65 & 6.1 \\
\hline & -1.33 & 0.969 & 0.80 & 32.7 \\
\hline NGC 253-2 (one) & -3.40 & 0.910 & 2.47 & 12.8 \\
\hline \multirow[t]{2}{*}{ NGC 253-2 (two) } & -8.34 & 0.952 & 4.39 & 5.2 \\
\hline & -2.14 & 0.999 & 0.97 & 20.3 \\
\hline NGC 1068 (one) & -3.13 & 0.921 & 2.88 & 13.9 \\
\hline \multirow[t]{2}{*}{ NGC 1068 (two) } & -6.09 & 1 & 5.80 & 7.1 \\
\hline & -1.31 & 1 & 0.68 & 33.2 \\
\hline IC 342 (one) & -4.26 & 0.936 & 2.67 & 10.2 \\
\hline \multirow[t]{2}{*}{ IC 342 (two) } & -10.87 & 0.997 & 2.87 & 4.0 \\
\hline & -1.50 & 1 & 0.27 & 28.9 \\
\hline M82-NE (one) & -3.91 & 0.958 & 2.64 & 11.1 \\
\hline \multirow[t]{2}{*}{ M82-NE (two) } & -7.81 & 0.992 & 4.25 & 5.6 \\
\hline & -2.77 & 0.999 & 1.04 & 15.7 \\
\hline M82-SW (one) & -3.94 & 0.975 & 1.80 & 11.0 \\
\hline \multirow[t]{2}{*}{ M82-SW (two) } & -7.10 & 1 & 2.39 & 6.1 \\
\hline & -3.02 & 1 & 0.74 & 14.4 \\
\hline \multicolumn{5}{|l|}{ Size $=7^{\prime \prime}$} \\
\hline NGC 4038 (one) & -2.38 & 0.895 & 0.25 & 18.3 \\
\hline \multirow[t]{2}{*}{ NGC 4038 (two) } & -6.24 & 0.936 & 0.29 & 7.0 \\
\hline & -1.52 & 0.991 & 0.15 & 28.6 \\
\hline \multicolumn{5}{|l|}{ Size $=25 \% \times 7^{\prime \prime}$} \\
\hline NGC 4038 (one) & -2.43 & 0.891 & 0.42 & 17.9 \\
\hline \multirow[t]{2}{*}{ NGC 4038 (two) } & -6.49 & 0.943 & 0.51 & 6.7 \\
\hline & -1.48 & 0.993 & 0.24 & 29.2 \\
\hline \multicolumn{5}{|l|}{ Size $=50 \% \times 7^{\prime \prime}$} \\
\hline NGC 4038 (one) & -2.46 & 0.887 & 0.88 & 17.6 \\
\hline \multirow[t]{2}{*}{ NGC 4038 (two) } & -6.67 & 0.947 & 1.10 & 6.5 \\
\hline & -1.45 & 0.995 & 0.49 & 29.9 \\
\hline \multicolumn{5}{|l|}{ Size $=75 \% \times 7^{\prime \prime}$} \\
\hline NGC 4038 (one) & -2.47 & 0.885 & 0.34 & 17.6 \\
\hline \multirow{2}{*}{ NGC 4038 (two) } & -6.74 & 0.949 & 4.29 & 6.5 \\
\hline & -1.42 & 0.997 & 1.85 & 30.5 \\
\hline \multicolumn{5}{|l|}{ Variable size } \\
\hline NGC 4038 (one) & -1.90 & 0.885 & 0.36 & 22.9 \\
\hline \multirow[t]{2}{*}{ NGC 4038 (two) } & -5.40 & 0.962 & 0.34 & 8.0 \\
\hline & -1.16 & 0.928 & 0.26 & 37.5 \\
\hline NGC 4039 (one) & -3.21 & 0.847 & 0.81 & 13.5 \\
\hline \multirow{2}{*}{ NGC 4039 (two) } & -11.34 & 1 & 1.01 & 3.8 \\
\hline & -1.27 & 1 & 0.55 & 34.1 \\
\hline Overlap (one) & -5.67 & 1 & 0.07 & 7.7 \\
\hline M 83 (one) & -2.73 & 0.999 & 0.45 & 15.9 \\
\hline
\end{tabular}

Notes. The linear regressions have been obtained using the xmgrace software, including in the calculations the error bars of the observations.

a The total source-averaged CS column density is derived using the following formulae: $N(\mathrm{CS})=10^{\text {intersect }} \times Q_{\text {rot }}\left(T_{\text {rot }}\right)$, where "intersect" is the value of the intersection of the linear regression with the $y$-axis (see Figures 11-16) and $Q_{\mathrm{rot}}\left(T_{\mathrm{rot}}\right)$ is the partition function at the rotational temperature $T_{\text {rot }}$ (linear interpolation realized when the available range of $T_{\text {rot }}$ was not relevant for the studied case-see the text). The rotational temperature is calculated using the formulae: $T_{\text {rot }}=-\frac{1}{\text { slope }} \times \log (e)$.

agreement with that derived by Mauersberger et al. (1989) and Martín et al. (2009) when using their respective single transition of $\mathrm{CS}$.

In the Antennae Galaxies case, using CO data for representing the more compact (CS) emitting source size is misleading and can cause large uncertainties on the results as shown by
Helfer \& Blitz (1995) for NGC 1068. Taking NGC 4038 as a representative example, we have thus investigated the influence of the source size choice on the results (LTE and non-LTE models, see Section 3.2) in the following way.

1. We have compared the physical parameters of column density and temperature derived assuming a source extent of $7^{\prime \prime}$, with those obtained by assuming sizes of $75 \%$, $50 \%$, and $25 \%$ smaller (i.e., $5^{\prime \prime} .25,3^{\prime \prime} .5$, and $1^{\prime \prime} .75$ ) similar to all transitions. We have rebuilt consistently the rotational diagrams and re-calculated in the same way as previously described, the rotational temperature and the total CS column density values. When these values were compared to those obtained in the case of a $7^{\prime \prime}$ source size, we have obtained rotational temperatures difference less than $7 \%$ and column densities differences within a factor of $<14.8$ (for the smallest size considered), whatever the number of components considered (see Table 2).

2. We compared the result of a common $7^{\prime \prime}$ source size for all transitions with the case of a gradually smaller size for the higher transitions. Starting with a source size of $7^{\prime \prime}$ for the CS(2-1) transition, we assumed a $20 \%$ smaller size for the 3-2 and the 4-3 (i.e., 5".6) and an additionally $20 \%$ decrease in the size for the 5-4 and the 7-6 (i.e., 4..5). When comparing these cases with the case of a $7^{\prime \prime}$ source size applied homogeneously to all the CS lines, it resulted a $15 \%-30 \%$ difference in rotational temperatures and less than a factor of 2 in column density.

As shown in both cases (see Table 2), though the column densities might be significantly affected by the assumed source extent, both the derived rotational temperatures and therefore the different temperature components described in this paper will be basically unaffected.

In all the cases dealt with above, the CS line ratios used for constraining the LVG model do change by no more than a factor of 1.5 (for the $\mathrm{CS}(7-6) / \mathrm{CS}(4-3)$ line ratio). Considering the degeneracy of the LVG models, as noted in Section 3.2, we thus believe that these variations of line ratios are negligible and that the results presented in Table 3 still provide qualitative estimates of the properties of the gas emitting the CS lines.

\subsection{Non-LTE Models}

It is well known that rotational diagrams give only approximate estimates of the kinetic temperature and the column density. To get more accurate values, we have ran LVG models (Goldreich \& Kwan 1974; de Jong et al. 1975). These models are described in various papers and the version we use in this study is the one presented in Bayet et al. (2004, 2006, 2008a). The LVG model runs with three free parameters: the gas density $n\left(\mathrm{H}_{2}\right)$, the kinetic temperature $\left(T_{k}\right)$, and the CS column density divided by the line width $\left(N\left({ }^{12} \mathrm{CS}\right) / \Delta v\right)$. Using as model constraints the integrated line intensity ratios computed from Table 2, and corrected for beam dilution effect (as done in Section 3.1), we have investigated the following range of LVG input parameters: $5 \mathrm{~K}<T_{k}<150 \mathrm{~K}, 1 \times 10^{11} \mathrm{~cm}^{-2}\left(\mathrm{~km} \mathrm{~s}^{-1}\right)^{-1}<$ $N\left({ }^{12} \mathrm{CS}\right) / \Delta v^{9}<1 \times 10^{16} \mathrm{~cm}^{-2}\left(\mathrm{~km} \mathrm{~s}^{-1}\right)^{-1}$ and $1 \times 10^{4} \mathrm{~cm}^{-3}<$ $n\left(\mathrm{H}_{2}\right)<1 \times 10^{7} \mathrm{~cm}^{-3}$.

For determining the set of parameters $\left(T_{k}, n\left(\mathrm{H}_{2}\right), N\left({ }^{12} \mathrm{CS}\right)\right)$ able to reproduce best the observations, we have constrained the predicted CS line intensity ratios with the observed values via a reduced $\chi^{2}$ method as performed in Bayet et al. (2004,

\footnotetext{
9 We have assumed for all the sources and line profiles $\Delta v \approx 100 \mathrm{~km} \mathrm{~s}^{-1}$.
} 
Table 3

Results of the LVG Model Analysis: The Physical Properties of the Best LVG Model (Having the Lowest $\chi^{2}$ Value) for Both the Low- and High-temperature Gas Components

\begin{tabular}{lccc}
\hline \hline Source & $\begin{array}{c}T_{k} \\
(\mathrm{~K})\end{array}$ & $\begin{array}{c}n\left(\mathrm{H}_{2}\right) \\
\left(\times 10^{5} \mathrm{~cm}^{-3}\right)\end{array}$ & $\begin{array}{c}N(\mathrm{CS}) \\
\left(\times 10^{14} \mathrm{~cm}^{-2}\right)\end{array}$ \\
\hline NGC 253-1 & 30 & 0.40 & 2.50 \\
& 65 & 25.0 & 0.63 \\
\hline NGC 253-2 & 15 & 0.16 & 4.00 \\
& 70 & 10.0 & 1.00 \\
\hline NGC 1068 & 20 & 1.50 & 6.30 \\
\hline IC 342 & 65 & 40.0 & 0.63 \\
\hline M82-NE & 15 & 0.63 & 1.00 \\
& 50 & 16.0 & 0.25 \\
\hline M82-SW & 10 & 1.00 & 4.00 \\
& 65 & 6.30 & 1.00 \\
\hline NGC 4038 & 15 & 1.60 & 2.50 \\
\hline NGC 4039 & 45 & 6.30 & 0.63 \\
\hline Overlap & 10 & 1.00 & 0.25 \\
\hline M 83 & 50 & 40.0 & 0.16 \\
\hline
\end{tabular}

Note. We remind the reader that these values are only indicative since degeneracy in LVG models occurs.

2006, 2008a), including $20 \%$ uncertainties on the observed integrated line intensities. Except for M 83 and Overlap, we have separated the model results into two: the low-temperature component constrained by, for example, line intensity ratios such as $\mathrm{CS}(3-2) / \mathrm{CS}(2-1)$ or $\mathrm{CS}(4-3) / \mathrm{CS}(2-1)$, and the hightemperature component characterized by, for example, line intensity ratios such as $\mathrm{CS}(5-4) / \mathrm{CS}(4-3)$ or $\mathrm{CS}(7-6) / \mathrm{CS}(4-3)$. The choice of the CS line intensity ratios used for calculating the $\chi^{2}$ depends on the source and the available data set for CS (see Table 1). For M 83 and Overlap, since the CS data are well arranged alongside a line in the rotational diagram, only a single-temperature component model has been investigated using the available CS detections. The physical properties which reproduce best the observations are summarized in Table 3.

Unfortunately, as already shown by, e.g., Bayet et al. (2004, 2006) and Martín et al. (2005, 2006a), there is no unique solution for reproducing the observational line ratios of each gas component, in each galaxy. Indeed, it is well known that degeneracies in the physical parameter predictions exist in LVG models. To remove this degeneracy and obtain more accurate estimations of the physical parameters, one should correlate LVG model solutions obtained for various molecular species. However, this methodology is only effective if all species are emitted from the same gas phase (see, e.g., Martín et al. 2005, 2006a). For most of the sources observed here, no other tracers of very dense gas $\left(n\left(\mathrm{H}_{2}\right) \geqslant 10^{5-6}\right)$ have been detected so far. We thus did not cross correlate our LVG results with those from other molecular emission.

\section{DISCUSSION}

We find that CS is indeed tracing very dense gas. For most sources, low- $J$ and high- $J$ CS lines trace different components. The low-temperature component, on average over the sources, has a $T_{\text {rot }}<30 \mathrm{~K}, N(\mathrm{CS})>1.0 \times 10^{14} \mathrm{~cm}^{-2}$, and $n\left(\mathrm{H}_{2}\right)<$ $1.6 \times 10^{5} \mathrm{~cm}^{-3}$, while the high-temperature component has a $T_{\text {rot }}>45 \mathrm{~K}$ with $N(\mathrm{CS})<1.0 \times 10^{14} \mathrm{~cm}^{-2}$ and $n\left(\mathrm{H}_{2}\right)>$ $2.5 \times 10^{5} \mathrm{~cm}^{-3}$. For the brightest sources, we compared the physical properties of these two CS components with the physical properties derived from other tracers of dense gas, when available. For the M 82-NE and M 82-SW molecular lobes, the low-temperature CS component is in very good agreement with the low-temperature component of methanol (see Martín et al. 2006a). Indeed their predicted (from LVG) kinetic temperatures are in agreement within a factor of $\approx 2-3$, while the gas densities and the column densities are in agreement within a factor of $<3$ and $<2$, respectively. Similarly, the physical properties of the high-temperature component of the CS are in good agreement with those of the high-temperature component of the methanol. Hence, it may be that the warm and dense methanol gas (respectively cold and less dense methanol gas) might be well mixed with the warm and dense CS gas (respectively cold and less dense CS gas) in these two positions. We do not know if this correlation is common to all $\mathrm{PDR}^{10}$-dominated galaxies, or if it depends on the galaxy type. Unfortunately, in the literature, no sample of methanol lines as complete as the one provided by Martín et al. (2006a) for M 82 exists. Detections of methanol in another starburst-dominated galaxy, NGC 253, exist (see, e.g., Martín et al. 2006b) but the authors do not distinguish between NGC 253-1 and NGC 253-2 and do not provide LVG predictions. In the same vein, in pure AGN-dominated galaxies (such as NGC 1068) or in merging-dominated systems (such as the Antennae Galaxies), no large sample of methanol lines have been analyzed under LVG approximations so far.

Similarly to the molecular lobes of $\mathrm{M} \mathrm{82}$, in both NGC 253-1 and NGC 253-2, the low-temperature CS component shows physical properties in very good agreement with the properties derived for $\mathrm{HCN}$ (single-temperature component whose properties are derived using an LTE analysis; see Martín et al. 2006b). The rotational temperatures for $\mathrm{HCN}$ and $\mathrm{CS}$ in NGC 253-1 (and NGC 253-2) are in agreement within a factor of $<2$ (respectively $<1.4$ ), while the $\mathrm{HCN}$ and the CS column densities in NGC 253-1 (and NGC 253-2) agree within a factor of $<1.5$ (respectively $<2.9$ ). Similarly, in both NGC 253 sources, the high-temperature CS component shows physical properties in agreement with those derived from $\mathrm{HCO}^{+}, \mathrm{H}_{2} \mathrm{CO}$, and $\mathrm{HNC}$ (single-temperature component whose properties are derived using an LTE analysis; see Martín et al. 2006b). This shows that the $\mathrm{HCN}$ and the low-temperature $\mathrm{CS}$ gas on one side, and the $\mathrm{HCO}^{+}, \mathrm{H}_{2} \mathrm{CO}, \mathrm{HNC}$, and the high-temperature $\mathrm{CS}$ gas on the other side, form two distinguished groups.

Furthermore, it is of particular interest to compare CS and $\mathrm{HCN}$ data in NGC 1068 (AGN-dominated galaxy). For this source, Krips et al. (2008) presented a low-temperature HCN component from LVG modeling constrained by a reasonable sample of HCN lines. This HCN gas component has $T_{k}, n\left(\mathrm{H}_{2}\right)$, $N(\mathrm{CS})=20 \mathrm{~K}, 10^{4}-10^{4.5} \mathrm{~cm}^{-3}, 10^{15}-10^{16} \mathrm{~cm}^{-2}$ which is a less dense and higher column density component than either our low-temperature or our high-temperature CS component. However, taking into account the limitations and uncertainties of LVG models, it may not be appropriate to compare the two sets of data.

In parallel, for M 82-NE and M 82-SW molecular lobes, Table 1 shows a significant narrowing of the FWHM of the CS(7-6) lines, as compared with the FWHM of the lower-J CS transitions. This feature has already been observed and analyzed by Bayet et al. (2008a) in the nucleus position. They interpreted

\footnotetext{
10 PDR stands for "photon dominated regions".
} 
this characteristic as the presence of multiple gas components in the source, the $\mathrm{CS}(7-6)$ line tracing a gas component warmer and denser than the gas traced by lower-J CS lines. Population rotational diagrams as well as LVG analysis (see Sections 3.1 and 3.2) confirm that this feature also appears in the molecular lobes of M 82. However, such narrowing does not appear in the other sources. Observations of the $\operatorname{CS}(7-6)$ transition in a larger sample of galaxies are needed in order to reveal very dense gas properties in PDR-dominated galaxies.

\section{CONCLUSIONS}

In order to better determine the properties of the very dense star-forming gas over a large range of physical conditions, we present the most complete, extragalactic CS survey ever performed on nearby galaxies. In particular, we detect for the first time the CS(5-4) and CS(7-6) lines, in various environments such as starburst, AGN-dominated galaxies, irregular galaxies, merging galaxies, etc.

In this first paper out of a series, we simply analyzed the data through a rotational diagram method and an LVG modeling, and we show that for most sources at least two gas components are needed to reproduce the observations. The low-temperature gas component properties, derived from the CS(2-1), CS(3-2), and $C S(4-3)$ lines (low- $J$ CS lines), vary from source to source within the range $T_{k}, n\left(\mathrm{H}_{2}\right), N(\mathrm{CS})=10-30 \mathrm{~K},(0.16-1.60) \times$ $10^{5} \mathrm{~cm}^{-3},(0.25-6.30) \times 10^{14} \mathrm{~cm}^{-2}$, respectively. The hightemperature gas component properties, derived from the CS(54) and CS(7-6) lines (high- $J$ CS lines), vary from source to source within the range $T_{k}, n\left(\mathrm{H}_{2}\right), N(\mathrm{CS})=45-70 \mathrm{~K},(6.30$ $40.0) \times 10^{5} \mathrm{~cm}^{-3},(0.16-1.00) \times 10^{14} \mathrm{~cm}^{-2}$, respectively. We also show that, using the current data set of CS observations in Overlap and $M$ 83, it appears that the very dense gas may be more homogeneously distributed than in other nearby sources. However, we again underline here that our estimates of the physical properties of these galaxies suffer from LVG degeneracy.

Comparison with other tracers of dense gas such as $\mathrm{HCN}$, $\mathrm{HNC}, \mathrm{HCO}^{+}$, etc., even in the brightest sources of our sample is very difficult to perform. However, in the molecular lobes of $\mathrm{M}$ 82 , we have been able to show a good correlation between the CS and the methanol. In both NGC 253-1 and NGC 253-2, we showed that the $\mathrm{HCN}$ and the low-temperature $\mathrm{CS}$ gas on one side, and the $\mathrm{HCO}^{+}, \mathrm{H}_{2} \mathrm{CO}, \mathrm{HNC}$, and the high-temperature $\mathrm{CS}$ gas on the other side, have compatible rotational temperatures and column densities.

In pure AGN-dominated galaxy, such as NGC 1068, we find no correlation between the HCN and CS.

Forthcoming papers will focus on detailed studies of the very dense gas properties in individual nearby sources, investigating for instance their dense gas star formation efficiencies, their dense gas masses based on CS modeling, etc., as well as on a more statistical analysis such as Kennicutt-Schmidt laws in both extragalactic and galactic sources.

E.B. acknowledges financial support from the Leverhulme Trust and M. Banerji for her participation to one of the observa- tional sessions on the top of the Mauna Kea. The James Clerk Maxwell Telescope is operated by The Joint Astronomy Centre on behalf of the Science and Technology Facilities Council of the United Kingdom, the Netherlands Organization for Scientific Research, and the National Research Council of Canada. Authors acknowledge the anonymous referee for his/her useful comments which significantly improved the paper. R.A. acknowledges financial support by the Spanish Ministerio de Ciencia e Innovacíon under project AYA2008-06181-C02-02. This work has also been partially supported by the Spanish Ministerio de Ciencia e Innovacion under project ESP200765812-C02-01, and by the Comunidad de Madrid Government under PRICIT project S-0505/ESP-0237 (ASTROCAM).

\section{REFERENCES}

Bayet, E., Gerin, M., Phillips, T. G., \& Contursi, A. 2004, A\&A, 427, 45

Bayet, E., Gerin, M., Phillips, T. G., \& Contursi, A. 2006, A\&A, 460, 467

Bayet, E., Lintott, C., Viti, S., Martín-Pintado, J., Martín, S., Williams, D. A., \& Rawlings, J. M. C. 2008a, ApJ, 685, L35

Bayet, E., Viti, S., Williams, D. A., \& Rawlings, J. M. C. 2008b, ApJ, 676 978

Bronfman, L., Nyman, L.-A., \& May, J. 1996, A\&AS, 115, 81

de Jong, T., Dalgarno, A., \& Chu, S. -I. 1975, ApJ, 199, 69

García-Burillo, S., Martín-Pintado, J., Fuente, A., \& Neri, R. 2001, ApJ, 563 L27

García-Burillo, S., Martín-Pintado, J., Fuente, A., Usero, A., \& Neri, R. 2002, ApJ, 575, L55

Girart, J. M., Viti, S., Williams, D. A., Estalella, R., \& Ho, P. T. P. 2002, A\&A, 388, 1004

Goldreich, P., \& Kwan, J. 1974, ApJ, 189, 441

Goldsmith, P. F., \& Langer, W. D. 1999, ApJ, 517, 209

Greve, T. R., Papadopoulos, P. P., Gao, Y., \& Radford, S. J. E. 2009, ApJ, 692, 1432

Helfer, T. T., \& Blitz, L. 1995, ApJ, 450, 90

Klein, R. I., Sandford, M. T., II, \& Whitaker, R. W. 1983, ApJ, 271, L69

Krips, M., Neri, R., García-Burillo, S., Martín, S., Combes, F., Graciá-Carpio, J., \& Eckart, A. 2008, ApJ, 677, 262

Larosa, T. N. 1983, ApJ, 274, 815

Lintott, C., \& Viti, S. 2006, ApJ, 646, L37

Martín, S., Martín-Pintado, J., \& Mauersberger, R. 2006a, A\&A, 450, L13

Martín, S., Martín-Pintado, J., \& Mauersberger, R. 2009, ApJ, 694, 610

Martín, S., Martín-Pintado, J., Mauersberger, R., Henkel, C., \& García-Burillo, S. 2005, ApJ, 620, 210

Martín, S., Mauersberger, R., Martín-Pintado, J., García-Burillo, S., \& Henkel, C. 2003, A\&A, 411, L465

Martín, S., Mauersberger, R., Martín-Pintado, J., Henkel, C., \& García-Burillo, S. 2006b, ApJS, 164, 450

Mauersberger, R., \& Henkel, C. 1989, A\&A, 223, 79

Mauersberger, R., Henkel, C., Wilson, T. L., \& Harju, J. 1989, A\&A, 226, L5

Meier, D. S., Turner, J. L., Crosthwaite, L. P., \& Beck, S. C. 2001, AJ, 121, 740

Muraoka, K., et al. 2009, PASJ, 61, 163

Paglione, T. A. D., Jackson, J. M., Ishizuki, S., \& Rieu, N. 1995, AJ, 109, 1716

Peng, R., Zhou, S., Whiteoak, J. B., Lo, K. Y., \& Sutton, E. C. 1996, ApJ, 470, 821

Planesas, P., Scoville, N., \& Myers, S. T. 1991, ApJ, 369, 364

Plume, R., Jaffe, D. T., \& Evans, N. J., II 1992, ApJS, 78, 505

Schinnerer, E., Böker, T., Meier, D. S., \& Calzetti, D. 2008, ApJ, 684, L21

Turner, B. E. 1991, ApJS, 76, 617

Walker, C. E., Walker, C. K., Carlstrom, J. E., \& Martin, R. N. 1990, in The Interstellar Medium in External Galaxies, ed. D. J. Hollenbach \& H. A. Thronson, Jr. (NASA CP-3084; Washington, DC: NASA), 78

Wilson, C. D., Scoville, N., Madden, S. C., \& Charmandaris, V. 2000, ApJ, 542, 120 